\title{
Forensic engineering of advanced polymeric materials. Part III - Biodegradation of thermoformed rigid PLA packaging under industrial composting conditions
}

Marta Musioł ${ }^{a},{ }^{1}$ Wanda Sikorska ${ }^{a}$, Grazyna Adamus ${ }^{a}$, Henryk Janeczek ${ }^{a}$, Jozef Richert ${ }^{b}$, Rafal Malinowski ${ }^{c}$, Guozhan Jiang, ${ }^{d}$ Marek Kowalczuk ${ }^{a, d^{*}}$

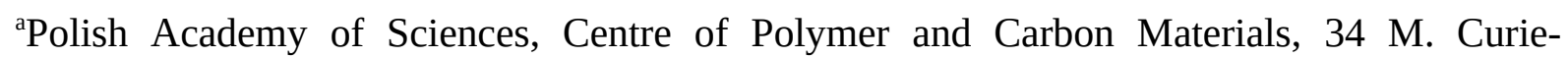
Sklodowska Str., 41-800 Zabrze, Poland; ' ${ }^{\text {} W W ~ E K O C H E M ~ L L C, ~} 1$ Akacjowa Str., 87-123 Dobrzejewice; 'Institute for Engineering of Polymer Materials and Dyes, 55 M. CurieSklodowska Str., 87-100 Torun; ${ }^{\mathrm{d}}$ University of Wolverhampton, Faculty of Science and Engineering, Department of Biology, Chemistry and Forensic Science, Wulfruna Street, Wolverhampton, WV1 1SB, UK

\section{ABSTRACT}

We report the forensic engineering study on the biodegradation behavior of a rigid packing tray thermoformed from PLA extruded foil as well as plain PLA foil under industrial composting and abiotic laboratory conditions. The hydrolytic degradation in water was conducted for reference. The effects of composting duration on the changes in molecular mass, glass transition temperature, and degree of crystallinity of the polymeric material were monitored using GPC and DSC. The chemical structure of dissolved degradation products of the polymeric material were examined using NMR and ESI-MS. The results show that the biodegradation process is less dependent on the thermal processing process of PLA and more on the applied composting/degradation conditions. There is an increase in dispersity, and the molecular mass distribution shows a bimodal profile, suggesting an autocatalytic hydrolysis effect during the ealry stage of the composting process. During the composting process, the

\footnotetext{
${ }^{1}$ Corresponding author. E-mail: m.musiol@cmpw-pan.edu.pl; Tel.: +48 32 271-60-77; Fax: +48 32 271-29-69

* Corresponding author. E-mail: M.Kowalczuk@wlv.ac.uk; Tel.: + 441902322272
} 
bulk hydrolysis mechanism operates dominantly. The PLA samples are also shown to have a gradual increase in opacity due to an increase in the degree of crystallinity.

\section{Keywords}

biodegradable polymers, polylactide (PLA), thermoformed rigid packaging, composting 


\section{INTRODUCTION}

Forensic engineering of advanced polymeric materials (FEAPM) deals with the evaluation and understanding of the relationships between their structure, properties and behavior before, during and after practical applications (Rydz et al. 2015, Sikorska et al., 2014). This new area of knowledge obeys the ex-ante investigations as well as the ex-post studies in order to increase efficiency and to define and minimize the potential failure of novel polymer products and provides basic knowledge for helping prevent future problems.

Biodegradable plastic packaging has become available on the market. This is due to a growing awareness of the pollution caused by the use of non-degradable plastics and difficulties in recycling stemmed from contaminations by foodstuffs, soil, water, chemicals, debris and other biological substance contents. The use of biodegradable plastics packaging will overcome these problems by composting, an organic recycling process that enables the removal of biodegradable plastic wastes through biological processes and allows the waste bioplastic products to participate the fundamental ecological material cycles. The stable compost formed from biodegradable packaging together with other components can be used for different purposes, e.g., in agriculture and horticulture as a fertilizer or as a plant growth substrate and for land re-cultivation (Abe et al.,1995).

Polylactide (PLA) is one of the commercially available biodegradable plastics used for manufacturing packaging. Its biodegradation is well understood through the extensive research in physiological environment due to their outstanding biocompatibility (Abe et al.,1995, Li McCarthy 1999, Chen et al., 2004, Zhang et al., 2008, Majid et al., 2002, Freier et al., 2002, Andersson et al., 2010a, 2010b, Yoshie et al., 2000, Mukai et al., 1993, Kunioka et al., 2006, Zenkiewicz et al., 2013, Arias et al., 2014 ). It is generally considered that bulk hydrolytic mechanism operates dominantly in the degradation of PLA. Enzymatic degradation is not convincing. The hydrolytic degradation has four stages: water absorption, cleavage of 
the ester bonds, diffusion of soluble oligomers and solubilization of fragments. When a thick sample is subjected to degradation, an autocatalytic process usually occurs since the interior will become more acidic due to the generation of more carboxylic acid group with the cleavage of the ester bonds, and these acidic groups are very slow to diffuse out of the material. In contrast, the carboxylic acids generated on the surface layer can leave the surface immediately. The semi-crystalline nature of PLA also affects the degradation behavior. The water diffused into the sample first cleaves the ester bonds in the amorphous regions. After most of the ester bonds in the amorphous region are broken down, the water starts to attack the crystalline regions. During degradation, water works as plasticizer to the PLA, enhancing the mobility of the polymer chains. The enhanced mobility leads to an increase in degree of crystallinity, resulting in loss of transparency of the plastics. At later stages of the degradation of PLA when the molecular weight is reduced significantly and fragmentation occurs, the presence of various enzymes can accelerate the degradation such as proteinsase $\mathrm{K}$, a serine protease from the fungus Tritirachium album (Reeve et al., 1994, Cai et al., 2005), lipase, esterase, and alcalase (Lee et al., 2014). Proteinase K preferentially degraded PLLA as opposed to PDLA (Reeve et al., 1994). Different enzymes have been shown a different degradation behavior. For example, alcalase is more efficient than lipase and esterase (Lee et al., 2014).

When PLA is used as a biodegradable packaging material, it is either discarded into the environment for degradation naturally or collected for composting. In a natural environment such as in soil burial and in aquatic system, the degradation is rather slow because the temperature is usually lower than $30^{\circ} \mathrm{C}$ (Rudnik, Brassoulis 2011) and very few PLAdegrading microorganisms distributed in the environment (Tokiwa et al., 2006). For example, in an environmental research report produced by The California Department of Resources Recycling and Recovery, the degradation of PLA was tested in a simulated marine 
environment for a period of six months at $25^{\circ} \mathrm{C}$. Only 3\% of PLA samples were degraded into $\mathrm{CO}_{2}$. The degradation of PLA is also very slow in many soil tests such as in natural Mediterranean soil (Rudnik, Brassoulis 2011) and in Costa Rican soil (Ho et al., 1999). After 11 months' burial test in Mediterranean soil at an average temperature of $21^{\circ} \mathrm{C}$ and $40 \%$ moisture, the mass loss is very small, although the mechanical properties and degree of crystallinity has a remarkable change.

Composting involves the conversion of a mixture of organic substances into $\mathrm{CO}_{2}, \mathrm{H}_{2} \mathrm{O}$ and humus under a biodegradation process by a microbial community composited of various populations in aerobic conditions and in solid phase. Composting conditions are quite different from environmental landfill because the latter lacks sufficient temperature, moisture, oxygen and microorganisms (Rudnik, Brassoulis 2011). Under landfill conditions, few bioplastics can meet the requirements of various testing standards such as ASTM 6400 used in the United States, DIN 54900 used in Germany, and EN 13432 used in most other European countries, in which the degradation is evaluated by the amount of $\mathrm{CO}_{2}$ release during the metabolic processes, where the polymer is used as a carbon source for microorganisms. At compost conditions, the enzymatic degradation may occur at later stages because of the presence of various microorganisms (W. Sikorska et al. 2015). Therefore, the degradation of PLA in compost conditions should be much more complex than degradation under physiological conditions or environmental conditions. These polyesters can be degraded enzymatically only after preliminary hydrolytic degradation (Penczek et al., 2013).

Composting of PLA has been studied in several reports under simulated conditions: in compost piles specially prepared for this purpose or in compost bins (Rudnik, Brassoulis 2011, Mohee et al., 2008). Kale et al. investigated the biodegradation of PLA spring water bottle, a tray and a deli container under real composting conditions $\left(\mathrm{T}>55^{\circ} \mathrm{C},>65 \%\right.$ relative 
humidity and $\mathrm{pH}$ 7.5). These packaging were subjected to composting test for 30 days (Kale et al., 2006). Rudnik et al. investigated the composting of PLA films and fibers at home composting conditions similar to soil burial (Rudnik, Brassoulis 2011). Ho et al. investigated the composting of PLA films. During the compost study, the temperature and relative humidity of the rows were maintained at 55 to $60^{\circ} \mathrm{C}$ and 50 to $70 \%$, respectively. A rapid loss of mechanical properties and molecular weight at first one or two weeks were observed in all the studies. A slight degree in glass transition temperature $\left(\mathrm{T}_{\mathrm{g}}\right)$ and melting temperature $\left(\mathrm{T}_{\mathrm{m}}\right)$ and an increase in crystallinity were also observed (Ho et al., 1999).

In the above composting studies of PLA, a film or thin sample was often used, and the biodegradation process was usually monitored using mechanical properties, molecular weight and thermal properties such as $\mathrm{T}_{\mathrm{g}}$ and $\mathrm{T}_{\mathrm{m}}$, and degree of crystallinity. Here we report the results of our study on biodegradation of PLA rigid foil and a thermoformed final packaging tray made from the foil under real industrial composting conditions. Two real industrial composting systems were used in the study: a static composting open-air pile and a KNEER system with containers. These two systems were selected in order to verify the influence of composting conditions on the biodegradation process. The influence of thermoforming process on biodegradation process was also examined. Additionally, the results of abiotic degradation under laboratory conditions were carried out in order to characterize degradation products for reference. The degradation was monitored using visual observation, molecular mass change, thermal property change and characterization of degradation products in order to understand the degradation process during industrial composting. 


\section{EXPERIMENTAL}

\subsection{Materials}

Polylactide used in this work was commercial PLA grade 2002 D (NatureWorks ${ }^{\circledR}$, USA) (Kolstad et al., 2012) with the following properties: $M_{w}=224000 \mathrm{~g} / \mathrm{mol}, M_{w} / M_{n}=1.9, T_{g}=$ $53.3{ }^{\circ} \mathrm{C}, T_{m}=147.4{ }^{\circ} \mathrm{C}, w_{c}=32.9 \%$. The rigid PLA foils made from the PLA for biodegradation tests were prepared by the IMPiB Institute in Torun, Poland (Bartczak et al., 2013). The test stand for extrusion flat foil was equipped with a single-screw extruder of a screw diameter of $45 \mathrm{~mm}$ and the length/diameter ratio equal to 37. The width of the extrusion die was $600 \mathrm{~mm}$. The stand was equipped with a set of calenders, an automatic foil thickness measurement system, take-off assembly and a winding device. Before processing the PLA was dried for $4 \mathrm{~h}$ at $75{ }^{\circ} \mathrm{C}$ to remove moisture to minimize degradation during extrusion. During extrusion the temperatures of the barrel heating zones I, II, III, IV and V and of the die of the extruder were set at $180,185,190,190,195$ and $200{ }^{\circ} \mathrm{C}$, respectively. The gear pump rotational speed was set at $100 \mathrm{rpm}$. The process leads to obtain transparent PLA foils $500 \mathrm{~mm}$ wide and $0.3 \mathrm{~mm}$ thick.

The foil was used to prepare a packaging product - trays as shown in Figure 1 with dimensions of $155 \times 120 \times 30 \mathrm{~mm}(\mathrm{~L} \times \mathrm{W} \times \mathrm{H})$. The foils were vacuum thermoformed on a stand with a negative form. During thermoforming the temperatures of heating zones were set at $240^{\circ} \mathrm{C}$ for all central zones and $280{ }^{\circ} \mathrm{C}$ for external zones. The holding time at lower pressure was $8 \mathrm{~s}$ and the the total duration of thermoforming was $45 \mathrm{~s}$. The cooling temperature of $50{ }^{\circ} \mathrm{C}$ was reached after approximately $15 \mathrm{~s}$. The dimensions of the form nest were $30 \times 120 \times 155 \mathrm{~mm}$, i.e. depth, width and length, respectively. 
Figure 1.

\subsection{Degradation experiments}

\subsubsection{Biodegradation under industrial composting conditions}

Biodegradation processes under industrial composting conditions (organic recycling) were performed at the Station of Mechanical-Biological Waste Treatment in Zabrze, Poland. Samples were incubated in a composting pile and in a KNEER container system that we described elsewhere (Musioł et al., 2011). KNEER is an intensive composting system with closed containers. The containers are connected to a biomass aeration system. Water during the process circulates in a closed cycle. The nascent gases are released to the atmosphere through a biological filter. A stage of intensive composting lasted for about 21 days. Composting pile contained $40 \%$ leaves, $30 \%$ branches and $30 \%$ grass, and in a container system contained 18\% leaves, 22\% branches, $23 \%$ grass and $37 \%$ collected domestic waste from selected segregation.

The samples were placed inside the steel cage and then the cage was filling up with compost from pile or container. The cages with the polymeric material were placed in a composting pile and in the container, at the depth of one meter under the compost surface. In the composting pile samples were incubated for 70 days at average temperature of $59{ }^{\circ} \mathrm{C}$. Experiments were conducted between July and August, with average daily rainfall below 0.1 $\mathrm{mm}$ and average daily temperature of $16.4{ }^{\circ} \mathrm{C}$ (http://stacje.katowice.pios.gov.pl/monitoring/). In the container system the samples were incubated for 21 days in three cages at average temperature of $60^{\circ} \mathrm{C}$.

\subsubsection{Abiotic degradation under laboratory conditions}


For abiotic degradation experiments samples (5 $\mathrm{cm} \mathrm{x} 1 \mathrm{~cm}$ strips) with average mass of $0.25 \mathrm{~g}$ were first dried under vacuum at room temperature to a constant mass to eliminate ultimate water content and were then incubated in screw-capped vials with air-tight PTFE/Silicone septa, containing $25 \mathrm{ml}$ of distilled water. The degradation experiment was being conducted for over 70 days at $70^{\circ} \mathrm{C}\left( \pm 0.5^{\circ} \mathrm{C}\right)$ as was described elsewhere (Sikorska et al., 2012).

The temperature was selected according to the accelerated degradation test conditions of ISO 15814:1999 standard . After a predetermined degradation time, the samples were separated from the degradation medium, washed with distilled water and dried under vacuum at room temperature to a constant mass. All tests were performed with triplicate measurements.

\subsection{Characterization of the samples before and after degradation tests}

\subsubsection{Visual examination}

The surface of the materials was done using Zeiss optical microscope (Opton-Axioplan) equipped with a Nikon Coolpix 4500 colour digital camera.

\subsubsection{Gel Permeation Chromatography (GPC) analysis}

The weight average molar mass $\left(\mathrm{M}_{\mathrm{w}}\right)$ of the PLA samples was determined by GPC experiments conducted in chloroform solution at $35{ }^{\circ} \mathrm{C}$ and a flow rate of $1 \mathrm{~mL} / \mathrm{min}$ using a Spectra-Physics 8800 solvent delivery system with two Mixed C Styragel columns in series and a Shodex SE 61 refractive index detector. Sample solutions in $\mathrm{CHCl}_{3}(10 \mu \mathrm{l}, 0.5 \% \mathrm{w} / \mathrm{v})$ were injected into the system. The GPC was calibrated using Polystyrene narrow standards.

\subsubsection{Differential scanning calorimetry (DSC)}


Thermal characteristics of the received materials were determined using the TA DSC 2010 apparatus (TA Instruments, New Castle, DE) at a heating rate of $10^{\circ} \mathrm{C} / \mathrm{min}$, in the range of 80 - $+200{ }^{\circ} \mathrm{C}$, with a nitrogen flow rate of $50 \mathrm{~mL} / \mathrm{min}$. The instrument was calibrated with indium and gallium standards. The melting temperature $\left(\mathrm{T}_{\mathrm{m}}\right)$ was taken as the temperature of the melting endotherm maximum and the glass transition temperature $\left(T_{g}\right)$ as the midpoint of the specific heat step associated with the transition.

\subsubsection{ESI-MS analysis}

Electrospray ionization mass spectrometry (ESI-MS) analysis was performed using a Finnigan LCQ ion trap mass spectrometer (Finnigan, San Jose, CA, USA). PLA samples were dissolved in a water/methanol system $(2: 1 \mathrm{v} / \mathrm{v})$ and the solutions were introduced into the ESI source by continuous infusion using a syringe pump at a rate of $3 \mu \mathrm{L} / \mathrm{min}$. The ESI source was operated at $4.5 \mathrm{kV}$, and the capillary heater was set to $200{ }^{\circ} \mathrm{C}$. Nitrogen was used as the nebulizing gas.

\subsubsection{NMR measurements}

Nuclear magnetic resonance (NMR) ${ }^{1} \mathrm{H}$ spectra were recorded using a Bruker-Advance spectrometer operating at $600 \mathrm{MHz}$ with Bruker TOPSPIN 2.0 software using $\mathrm{CDCl}_{3}$ as the solvent and tetramethylsilane (TMS) as the internal standard. Each spectrum was collected with 64 scans, $11 \mu$ s pulse width, and 2.65 s acquisition time.

\section{Results and discussion}

\subsection{Biodegradation under industrial composting conditions}


The PLA rigid foil as well as the thermoformed tray was subjected to the biodegradation tests under industrial composting conditions: in a composting pile and in a container. Figure 2 shows the macroscopic and microscopic pictures of the final product after 70 days of incubation in the composting pile.

\section{Figure 2.}

It can be seen from the macroscopic pictures that the studied materials become disintegrated during the degradation test after 70 days. The fragments become turbid from original transparence, which suggest an increase in their crystallinity during degradation process, as demonstrated in the following thermal analysis. The changes in molecular masses of the PLA rigid tray and the foil with incubation time in composting pile are shown in Figure 3. The two samples have quite similar degradation profile. After 14 days of incubation, the molecular masses of both the rigid tray and the rigid foil dropped to about half of the original molecular masses. This indicates that the degradations of the materials are comparable for the same type of composting system despite different processing they underwent (Sikorska et al., 2012).

Figure 3.

The molecular mass distribution of the material after degradation is shown in Figure 4. The PLA has a bimodal molecular mass distribution after incubation under industrial composing conditions. The formation of the distribution is most probably caused by the sufficient thickness of the sample, which leads to a heterogeneous degradation of the materials caused by the absorption of water during their incubation (Fig 3) (Grizzi et al., 1995). This is consistent with the general hydrolysis pattern of thick PLA samples. The diffused water degrades the polymer molecules of the interior of the materials, generating a significant amount of carboxylic acid groups which are difficult to diffuse out in contrast to those in the 
surface phase. The higher concentration of acidic groups results in a higher degradation rate for the interior part than the exterior part. A bimodal distribution may thus be formed.

Figure 4.

The absorption of water and the bimodal distribution of the GPC obtained for the PLA rigid foil and the final product indicate that the autocatalytic effect during the first step of the degradation process under industrial composting conditions occurs.

The influence of the type of composting systems on the degradation was shown in Figure 5, in which the retaining molecular masses of the samples were plotted against incubation time in the two different composting systems. The molecular mass has a faster degradation rate in the closed container system than in the open composting pile. After 20 days incubation in the closed container, the molecular mass of the sample decreased to about $1 / 5$, while it took about 50 days for a similar degradation in the open composting pile.

Figure 5.

\subsection{Abiotic degradation in laboratory conditions}

A hydrolytic degradation study at $70{ }^{\circ} \mathrm{C}$ was performed as a reference for the composting process (Sikorska et al., 2012). Before the test all materials were transparent. After 3 days of incubation the initially transparent samples became turbid, which is indicative of an increase in crystallinity during the degradation process as demonstrated in the following thermal analyses. At the same time cracks appeared on the samples' surface. Disintegration of the samples was observed after seven days of incubation. Figure 6 shows the GPC elugrams of the thermoformed tray after incubation in water for various durations at $70{ }^{\circ} \mathrm{C}$. 
Figure 6.

There is a clear bimodal molar mass distribution for incubation in water for 7 days and 14 days. The first peak of the distribution becomes weaker for 14 day's incubation. However, the first peak does not appear for an incubation period of 21 days. Since the formation of cracks and fragmentation of the sample after 7 days incubation, the diffusion of acidic compounds out of the interior becomes easy. The autocatalytic effect due to the thickness becomes more and more weak and finally disappears. Hence, the molar mass distribution changes from bimodal distribution to single peak distribution as shown in Figure 6.

For comparison, the changes in molar mass with incubation time for both PLA rigid foil and the thermoformed tray are shown in Figure 7. It can be seen that the degradation profiles of the two types of samples are quite similar. This indicates that thermal processing does not have a significant influence on the degradation behavior of PLA, which is consistent with the proceeding degradation behavior in industrial composting conditions. Similar results for the lack of influence of the number of the processing repetitions on the degradation rate of PLA were reported recently (Sikorska et al., 2012, Zenkiewicz el al. 2009).

\section{Figure 7.}

Figure 8 shows an example of ESI-MS spectra of the water medium in which the PLA rigid foil were incubated for the 21 days. There are two peaks in the ESI-MS spectra. The signal at $\mathrm{m} / \mathrm{z}=89.3$ is designated as lactic acid, and the signal at $\mathrm{m} / \mathrm{z}=161$ is designated as the dimer of lactic acid.

Figure 8. 
The bimodal distribution of the GPC elugrams of the materials and the presence of lactic acid and its dimer in degradation medium water indicate that during early stage of hydrolysis process, the autocatalytic effect occurs. The water-soluble oligomers which are immobilized in the matrix will be released into the degradation medium at later stages of degradation, when the molar mass of the degradation products drops to a low value, as demonstrated in our previous work (Sikorska et al., 2012). Because of the low concentration, degradation products were not detected by NMR spectroscopy before the $70^{\text {th }}$ day of incubation. Figure 9 shows the ${ }^{1} \mathrm{H}$ NMR spectrum of the degradation products of PLA rigid film after 70 days of incubation in water medium. The signals present in the spectrum can be assigned to the protons of the methyl group of the lactic acid and its dimer (Wang et al., 2005), which further confirms that the final degradation products are lactic acid and the dimer of lactic acid.

Figure 9.

\subsection{Thermal behavior of PLA degradation}

Loss of sample transparency was noticed during degradation under industrial composting conditions as well as in water at $70{ }^{\circ} \mathrm{C}$. In order to verify if the observed change can be attributed to the increase of crystallinity, a series of DSC measurements were performed. Figure 10 shows the DSC traces of the PLA rigid foil sample before and after 7 days of incubation in the composting pile and in water at $70{ }^{\circ} \mathrm{C}$.

\section{Figure 10.}

In the first calorimetric trace for the PLA rigid foil sample before incubation, the structural relaxation overlapped with the glass transition relaxation. The glass transition temperature $\left(\mathrm{T}_{\mathrm{g}}\right.$ ) of the amorphous sample was obtained from the third calorimetric trace (cooling run) in which this transition temperature is the only phenomenon shown, because the applied cooling 
rate $10^{\circ} \mathrm{C} / \mathrm{min}$, does not allow the crystallization. To obtain the glass transition temperature for PLA rigid foil sample after 7 days of incubation in water at $70{ }^{\circ} \mathrm{C}$, where the applied cooling rate allows crystallisation, the amorphous samples of PLA were first obtained by quenching from the melt. The glass transition temperature $\left(T_{g}\right)$ was next obtained from the heating run at $20^{\circ} \mathrm{C} / \mathrm{min}$ in which this is the only phenomenon revealed (Table 2).

The first heating DSC scan for the PLA rigid foil sample before incubation (Fig 10 a) does not exhibit a melting peak, suggesting that the sample is amorphous before the degradation test, as evidenced by DSC. The first heating DSC scan for the PLA rigid foil sample after 7 days of incubation in water at $70{ }^{\circ} \mathrm{C}$ (Fig $\left.10 \mathrm{~b}\right)$ shows melting at $138.1^{\circ} \mathrm{C}(\Delta H=61.7 \mathrm{~J} / \mathrm{g})$ and after 7 days of incubation in the composting pile (Fig $10 \mathrm{c}$ ) at $156.4{ }^{\circ} \mathrm{C}(\Delta H=33.9 \mathrm{~J} / \mathrm{g})$. The approximate degrees of crystallinity for sample PLA rigid foil after degradation were calculated as we described elsewhere (Sikorska et al., 2012) and are presented in Table 1.

Table 1.

These DSC results confirm that the loss of sample transparency is due to the crystallization process. The increase in crystallinity of PLA rigid foil samples could be associated with the hydrolytic degradation process that occurs in a privileged way in the amorphous regions of the sample (Hakkarainen et al., 1996). It is well known that the $\mathrm{T}_{\mathrm{g}}$ of a polymer depends on the length of the polymer chains (Montserrat, Colomer 1984). The decrease of the $T_{g}$ during degradation, especially in water at $70{ }^{\circ} \mathrm{C}$, is correlated with the decrease of molar mass.

During the first heating run of the PLA rigid foil after 7 days of incubation in the composting pile, a double melting endotherm were observed. Multiple melting peaks in the DSC curves have been reported for many semicrystalline polymers. In our case it could be a consequence 
of the degradation mechanism, which can lead to the formation of a dual population of crystallites (Rydz et al., 2013, Wang, Mano 2005).

\section{CONCLUSION}

In this work the FEAPM studies on rigid packing tray thermoformed from PLA extruded foil as well as plain PLA foil under industrial composting and abiotic laboratory conditions have been performed . The following conclusions can be drawn from the work:

- The thermoformed products and extruded rigid foil of PLA have similar degradation profile regardless industrial composting conditions and under abiotic degradation in water. Thermal processing has little effect on the degradation behavior of PLA.

- The degradation of PLA in closed container is much faster than in composting pile, and the degradation in $70^{\circ} \mathrm{C}$ water is much faster than in closed container. This indicates that the control of moisture and temperature is important factors that affect the degradation of PLA.

- The molecular mass distribution of PLA in the two industrial composting conditions and in water all show a bimodal profile, which is due to the autocatalytic effect of hydrolysis degradation. Therefore, hydrolysis mechanism operates dominantly in the composting. At later stage of degradation, the lactic acid and its dimer diffuse out of the interior of the samples, and the effect of autocatalytic degradation disappears.

- With an increase in the extent of degradation, the degree of crystallinity increases due to the decrease in molecular mass of the polymer, which results in the loss of transparency.

- The biodegradation test under industrial composting conditions may be considered as the "in vivo" test because it reflects the actual conditions prevailing during the course 
of organic recycling. The results suggest that the PLA thermoformed rigid packaging is compostable.

\section{ACKNOWLEDGEMENT}

The research was supported by the European Regional Development Fund: Contract no. POIG.01.03.01-00-018/08. The outcomes of this research will be used to implement the PLASTiCE project (3CE368P1, "Innovation value chain development for sustainable plastics in central Europe”, Central Europe Programme, co-financed by ERDF).

\section{Reference}

Abe, H., Matsubara, I., Doi, Y., 1995. Physical properties and enzymic degradability of polymer blends of bacterial poly[(R)-3-hydroxybutyrate] and poly[(R,S)-3hydroxybutyrate] stereoisomers. Macromolecules. 28, 844-853.

Andersson, S., Hakkarainen, M., Inkien, S., Sodergard, A., Albertsson, A-C., 2010 a. Polylactide stereocomplexation leads to higher hydrolytic stability but more acidic hydrolysis product pattern. Biomacromolecues. 11, 1067-1073.

Andersson, S., Hakkarainen, M., Albertsson, A-C., 2010 b Tuning the polylactide hydrolysis rate by plasticizer architecture and hydrophilicity without introducing new migrants. Biomacromolecules. 11, 3617-3623.

Arias, V., Höglund, A., Odelius, K., Albertsson, A-C., 2014. Tuning the degradation profiles of poly(L-lactide)-based materials through miscibility. Biomacromolecules. 15, 391402.

Bartczak, Z., Gałęski, A., Kowalczuk, M., Sobota, M., Malinowski, R., 2013. Tough blends of poly(lactide) and amorphous poly([R,S]-3-hydroxy butyrate) - morphology and properties. Eur Polym J. 49, 3630-3641.

Cai H., Dave V., Cross R. A., McCarthy S.P., 1996. Effects of Physical Aging, Crystallinity, and Orientation on the Enzymatic Degradation of Poly( Lactic acid). J. Polym. Sci., Part B: Polym. Phys. 34, 2701-2708

Chen, Y., Jia, Z., Schaper, A., Kristiansen, M. 2004. Hydrolytic and enzymatic degradation of liquid-crystalline aromatic/aliphatic copolyesters. Biomacromolecules. 5, 11-16.

Freier, T., Kunze, C., Nischan, C., Kramer, S., Sternberg, K., Saß, M., Hopt, U.T., Schmitz, K-P. 2002. In vitro and in vivo degradation studies for development of a biodegradable patch based on poly(3-hydroxybutyrate). Biomaterials. 23, 2649-2657. 
Grizzi, I., Garreau, H., Li, S., Vert, M. 1995. Hydrolytic degradation of devices based on poly(DL-lactic acid) size dependence. Biomaterials. 16, 305-311.

Hakkarainen, M., Albertsson, A-C., Karlsson, S. 1996. Weight losses and molecular weight changes correlated with the evolution of hydroxyacids in simulated in vivo degradation of homo- and copolymers of PLA and PGA. Polym Degrad Stab 52, 283291.

Ho, K.-L. G., Pometto III , A.L., Gadea-Rivas, A., Briceño, J.A., Rojas, A. 1999. Degradation of Polylactic Acid (PLA) Plastic in Costa Rican Soil and Iowa State University Compost Rows. J Environ Polym Degr 7, 173-177.

Kale, G., Auras, R., Singh, S.P. 2006. Degradation of commercial biodegradable packages under real composting and ambient exposure conditions. J Polym Environ. 14, 317334.

Kolstad, J., Vink, E., de Wilde, B., Debeer, L. 2012. Assessment of anaerobic degradation of Ingeo $^{\mathrm{TM}}$ polylactides under accelerated landfill conditions. Polym Degrad Stab 97, 1131-1141.

Kunioka, M., Ninomiya, F., Funabashi, M. 2006 Biodegradation of poly(lactic acid) powders proposed as the reference test materials for the international standard of biodegradation evaluation methods. Polym Degrad Stab. 91, 1919-1928.

Lee, S. H., Kim, Y.J., Song, W.S., 2014. Biodegradation of Polylactic Acid (PLA) Fibers Using Different Enzymes. Macromolecular Research, 22, 657-663.

Li, S., McCarthy, S., 1999. Further investigations on the hydrolytic degradation of poly (DLlactide). Biomaterials. 20, 35-44.

Majid, M.I.A., Ismail, J., Few, L.L., Tan, C.F. 2002. The degradation kinetics of poly(3hydroxy butyrate) under non-aqueous and aqueous conditions. Eur Polym J 38, 837839.

Mohee, R., Unmar, G.D., Mudhoo, A., Khadoo, P., 2008. Biodegradability of biodegradable/degradable plastic materials under aerobic and anaerobic conditions. Waste Manage 28, 1624-1629.

Montserrat, S., Colomer, R.,1984. The effect of the molecular weight on the glass transition temperature in amorphous poly(ethylene terephthalate). Polym Bull. 12, 173-180.

Mukai, K., Yamada, K., Doi, Y., 1993. Kinetics and mechanism of heterogeneous hydrolysis of poly[(R)-3-hydroxybutyrate] film by PHA depolymerases. Int J Biol Macromol 15, 361-366.

Musioł, M., Rydz, J., Sikorska, W., Rychter, P., Kowalczuk, M. 2011. A preliminary study of the degradation of selected commercial packaging materials in compost and aqueous environments. Pol J Chem Technol. 13, pp. 55-57. 
Penczek, S., Pretula, J., Lewiński, P., 2013. Polymers from renewable resources. Biodegradable polymers. Polimery 11-12, 833-958.

Reeve, M.S., McCarthy, S.P., Downey, M.J., Gross, R.A. 1994. Polylactide stereochemistry: effect on enzymic degradability. Macromolecules 27, 825-831.

Rudnik, E., Briassoulis, D. 2011. Degradation behaviour of poly(lactic acid) films and fibres in soil under Mediterranean field conditions and laboratory simulations testing. Ind Crop Prod. 33, 648-658.

Rydz, J., Wolna-Stypka, K., Adamus, G., Janeczek, H., Musioł, M., Sobota, M., Marcinkowski, A., Kržan, A., Kowalczuk, M. 2015. Forensic engineering of advanced Polymeric materials. Part 1 - Degradation Studies of Polylactide blends with atactic poly [(R, S) -3-hydroxybutyrate] in paraffin.Chem. Biochem. Eng. Q. 29, 247259.

Rydz, J., Wolna-Stypka, K., Musioł, M., Szeluga, U., Janeczek, H., Kowalczuk, M. 2013. Further evidence of polylactide degradation in paraffin and in selected protic media. A thermal analysis of eroded polylactide films. Polym Degrad Stab. 98, 1450-1457.

Sikorska, W., Adamus, G., Dobrzynski, P., Libera, M., Rychter, P., Krucinska, I., Komisarczyk, A., Cristea, M., Kowalczuk, M. 2014. Forensic engineering of advanced polymeric materials. Part II: The effect of the solvent-free non-woven fabrics formation method on the release rate of lactic and glycolic acids from the tin-free poly (lactide-co-glycolide) nonwovens. Polym Degrad Stab. 110, 518-528

Sikorska, W., Musiol, M., Nowak, B., Pajak, J., Labuzek, S., Kowalczuk, M., Adamus, G., 2015. Degradability of polylactide and its blend with poly[(R,S)-3-hydroxybutyrate] in industrial composting and compost extract. Int Biodeterior Biodegradation.101, 3241.

Sikorska, W., Richert, J., Rydz, J., Musioł, M., Adamus, G., Janeczek, H., Kowalczuk, M. 2012. Degradability studies of poly(L-lactide) after multi-reprocessing experiments in extruder. Polym Degrad Stab. 97, 1891-1897.

Tokiwa, Y., Calabia, B.P. 2006. Biodegradability and biodegradation of poly(lactide). Appl. Microbiol. Biotechnol.. 72, 244-251

Wang, Y., Mano, J.F. 2005. Influence of melting conditions on the thermal behavior of poly(L-lactic acid). Eur Polym J . 41, 2335-2342.

Yoshie, N., Nakasato, K., Fujiwara, M., Kasuya, K., Abe, H., Doi, Y., Inoue Y. 2000. Effect of low molecular weight additives on enzymatic degradation of poly(3hydroxybutyrate). Polymer . 41, 3227-3234.

Zhang, X., Espiritu, M., Bilyk, A., Kurniawan, L. 2008. Morphological behaviour of poly(lactic acid) during hydrolytic degradation. Polym Degrad Stab. 93, 1964-1970. 
Żenkiewicz, M., Richert, J., Rytlewski, P., Moraczewski, K., Stepczyńska, M., Karasiewicz, T., 2009. Characterisation of multi-extruded poly(lactic acid). Polym Test. 28, 412418.

Żenkiewicz, M., Richert, A., Malinowski, R., Moraczewski, K. 2013. A comparative analysis of mass losses of some aliphatic polyesters upon enzymatic degradation. Polym Test. 32, 209-214.

\section{Table}

Table 1. Calorimetric parameters during the degradation process in different environments

\section{Figure Captions}

Figure 1. Thermoformed final product (tray) prepared from PLA rigid foil: a - macroscopic, b - microscopic (120x)

Figure 2. Digital photographs of the thermoformed final product from PLA rigid foil after 70 days of degradation in composting pile: $\mathrm{a}$ - macroscopic, $\mathrm{b}$ - microscopic (120x)

Figure 3. The change in weight average molar mass of the final product and PLA rigid foil, during incubation in the container.

Figure 4. The GPC elugrams of the PLA rigid foil after 7 days of incubation in the container system.

Figure 5. The change in weight average molar mass of the final product, during incubation in the container and composting pile [\%]

Figure 6. The GPC elugrams of the thermoformed tray after a specific time of incubation in water at $70^{\circ} \mathrm{C}$.

Figure 7. The change in weight average molar mass of the final product and PLA rigid foil, during incubation in water at $70^{\circ} \mathrm{C}$.

Figure 8. A typical ESI-MS spectra in the range $\mathrm{m} / \mathrm{z} 50-200$ (negative ions) of the watersoluble degradation products of PLA rigid film after 21 days of incubation in water at $70{ }^{\circ} \mathrm{C}$

Figure 9. The ${ }^{1} \mathrm{H}$ NMR spectrum of the degradation products of PLA rigid foil after 70 days of incubation in water at $70^{\circ} \mathrm{C}-$ a zoomed in section in the range of $1.30-1.76 \mathrm{ppm}$.

Figure 10. The first DSC heating trace for PLA rigid foil sample (a) before the test, (b) after 7 days incubation in water at $70^{\circ} \mathrm{C}$ and (c) after 7 days incubation in composting pile 